\title{
La dimensión fronteriza de la política exterior de Chile: inmovilidad y emergencia de nuevas dinámicas ${ }^{1}$ \\ Cristian Ovando Santana* \\ Gonzalo Álvarez Fuentes**
}

\section{Resumen}

El documento constata las prioridades de la política pública fronteriza que define la política exterior de Chile. A través de un esquema teórico proveniente del debate racionalistareflexivo de las relaciones internacionales, se sostiene que pese a la creciente apertura, internacionalización del país y la emergencia de actores e iniciativas subnacionales en el ámbito fronterizo, no siempre coinciden las miradas y opciones entre los actores de este nuevo ámbito y las definiciones de política exterior. Se plantea la necesidad de establecer mecanismos que concilien las posturas entre actores políticos y sociales regionales con organismos intermedios y los provenientes del nivel central.

Palabras clave: constructivismo, política exterior, fronteras, Chile, Bolivia.

\begin{abstract}
The following article draws the priorities of border public policy that define the Chilean foreign policy. Through a theoretical framework from the rational-reflective approach of International Relations, we argue that despite of the increasing openness, internationalization of the country, and the emergence of new actors and subnational initiatives; the views and options between the actors of this new area and the definitions of foreign policy not always match. We propose the need to establish mechanisms that settle the positions among regional socialpolitical actors, intermediate agencies and the central level.
\end{abstract}

Keywords: constructivism, foreign policy, borders, Chile, Bolivia.

* Licenciado en Ciencias Políticas y Gestión Pública, Máster en Relaciones Internacionales. Académico del Instituto de Estudios Internacionales de la Universidad Arturo Prat. Correo electrónico: covando@unap.cl

** Licenciado y Magíster en Ciencia Política. Académico en la carrera de Estudios Internacionales de la Universidad de Santiago de Chile y del Programa de Bachillerato de la Universidad Diego Portales. Correo electrónico: gonzalo.alvarez.f@ usach.cl

${ }^{1}$ Resultado del proyecto "Diagnóstico de la dinámica transfronteriza chileno-boliviana presente en la región de Tarapacá: aproximaciones desde los actores claves”, Código Proyecto Di.0066-09. Financiado por la Dirección General de Investigación de la Universidad Arturo Prat, Iquique, Chile. 


\section{Introducción}

El objetivo de este trabajo apunta a determinar cuáles son las prioridades de la política pública fronteriza que define la política exterior de Chile, específicamente la referida a los territorios que comprenden la frontera que comparte con Bolivia, y cuáles son las principales divergencias y disparidades entre los distintos actores que intervienen en el proceso que pone en práctica esta política.

A partir de un caso concreto que manifiesta el incipiente desarrollo transfronterizo presente en la región de Tarapacá, ${ }^{2}$ el proyecto "Trinacional Aymaras Sin Fronteras", y su implementación en coordinación con actores nacionales, nos centramos en la trama de relaciones que surge entre los actores que diseñan la política exterior de Chile, que tienen incidencia en los territorios fronterizos, y los que la implementan.

Para adentrarnos en la temática, consideramos el contexto regional en el que se insertan dichas políticas, signado por una creciente interdependencia, la apertura hacia una nueva agenda de política exterior y la presencia esporádica en la agenda regional de avances y estancamientos en el despliegue de las políticas llevadas que afectan el desarrollo de las zonas fronterizas, así como en los estilos diplomáticos y las características de la política exterior chilena. Considerando el debate racionalista-reflexivo de las relaciones internacionales (RRII), se advierte una disparidad en las opciones de políticas públicas fronterizas y el diseño de la política exterior, denotado por los siguientes elementos:

- Primero, la emergencia de un discurso y práctica local que reivindica el desarrollo transfronterizo presente, por ejemplo, entre regiones del Norte Grande de Chile, el sur del Perú y el centro-oeste boliviano. Se trata de una opción concreta de iniciativas internacionales subnacionales que ya manifiesta algunos resultados visibles, sobre todo en el ámbito

${ }^{2}$ La Región de Tarapacá está ubicada a 1,800 kms al norte de la capital de Chile, Santiago; consta de dos provincias, Iquique y Tamarugal; limita al norte con la Región de Arica y Parinacota, al sur con la Región de Antofagasta, al este con la República de Bolivia y al oeste con el océano Pacífico. 
del municipalismo ${ }^{3}$ y la cooperación descentralizada (Rouviere, 2009; González, et al. 2008). Estos procesos se ven reforzados por dos tendencias presentes en la región: una, referida a las reformas de descentralización que han permitido un mayor despliegue de los actores locales en la esfera internacional (Santos, 2010); otra, vinculada a la aparición de actores de distinto ámbito en la acción pública local que amplía su margen de maniobra, lo que les permite hacer usos múltiples de su papel políticoinstitucional (Rouviere, 2007). Entre aquellos se encuentran estrategias de desarrollo internacional e integración subregional paralelas a las dinámicas interestatales, es el caso de las prácticas paradiplomáticas presentes en la región (Aranda et al., 2010; González, 2006). No obstante, pese a que estas nuevas prácticas se inscriben dentro de formas de adaptación a los cambios del entorno político y económico internacional, la tendencia actual en América Latina, desde las prioridades de las políticas exteriores, está signada por la ausencia de una política de desarrollo territorial que acompañe a las entidades territoriales en el proceso de la nueva inserción internacional (Botero-Ospina y Cardozo, 2009:615). Así, la prescindencia de procesos sociales y económicos con lógicas territoriales por parte de las políticas exteriores centrales (Debuyst, 2009; Botero-Ospina y Cardozo, 2009; Moncayo, 2002), sería uno de los principales escollos que afectarían las iniciativas de los actores subestatales presentes en el continente. Otro, haría referencia al despliegue de actividades internacionales, paralelas a los contenidos de la política exterior, cuestión que implica la falta de un marco jurídico y político-institucional que dé viabilidad y efectividad a estas iniciativas (Gallardo, 2007).

- Segundo, desde los organismos intermedios, como es la Subdere ${ }^{4}$ en el caso de Chile, se constata un decidido apoyo a estas iniciativas locales, fundamentalmente facilitando el desarrollo de proyectos, teniendo en cuenta el restringido marco jurídico que faculta emprender actividades

\footnotetext{
${ }^{3}$ Según datos que aporta la Subsecretaría de Desarrollo Regional de Chile (Subdere), los municipios pertenecientes a la región de Tarapacá emprenden actividades paradiplomáticas con 16 contrapartes. Dentro de sus áreas prioritarias la integración es la dimensión que más se repite en las distintas iniciativas (Subdere, 2009).

${ }^{4}$ Subsecretaría de Desarrollo Regional y Administrativo, dependiente del Ministerio del Interior. Dentro de sus tareas de coordinación, asesoramiento, dirección y ejecución de medidas para promover la descentralización del territorio, la Unidad de Rela-
} 
internacionales a actores subestatales (Tapia, 2003; Gallardo, 2007). Sus iniciativas han tendido a visibilizar la acción exterior de las regiones y comunas a través de colaboración técnica, sistematización y cuantificación de las actividades y gestiones emprendidas por distintos actores regionales y comunales. Adicionalmente, el organismo público ha planteado la necesidad de generar una mayor supervisión y coordinación por parte del gobierno central hacia las actividades internacionales emprendidas por las comunas, a objeto de mantener coherencia con la política exterior del país; para lo cual ha instado a la consideración de alguna modificación legal que permita el ordenamiento de la acción paradiplomática y de cooperación descentralizada en Chile a partir de la experiencia y práctica internacional (Subdere, 2008).

- Tercero, en cuanto al nivel central, a través de la Cancillería, las opciones apuntan a iniciativas concretas, referidas en su mayoría a mejorar los mecanismos de control fronterizo de flujos migratorios, controles aduaneros y fitosanitarios, en la medida en que son iniciativas funcionales a la estrategia de inserción comercial internacional emprendida por Chile en las últimas décadas. En menor grado, aunque con bastante presión por parte de los actores regionales, se impulsan acciones relacionadas con la optimización del transporte internacional e iniciativas como el corredor bioceánico. Estas medidas apuntan a disminuir la brecha entre el intercambio comercial promovido desde el nivel central y las especificidades y necesidades demandadas desde las regiones (Podestá, 2003:98). En cuanto a aquellos mecanismos de integración en que participan como miembros asociados Chile y Bolivia -como es el caso de la Comunidad Andina de Naciones y del Mercosur-, las políticas en términos de desarrollo fronterizo son muy débiles, dado que se focalizan más bien en las políticas macroeconómicas y macroterritoriales (Meza, 2005). Otro aspecto destacable surge desde que el incremento hacia políticas en el ámbito fronterizo de tendencia más territorial, estaría denotado por las fricciones diplomáticas presentes o ausentes con los países vecinos con que se emprenden estas iniciativas; de este modo,

ciones Internacionales de la Subdere apoya la inserción internacional de las regiones de Chile a través de la instalación y el fortalecimiento de la gestión internacional subnacional (ver: http://www.subdere.gov.cl/1510/w3-propertyvalue-28901.html). 
el aumento progresivo con pares argentinos, en comparación con lo alcanzado con los vecinos Perú y Bolivia, da cuenta de esta realidad. Por otro lado, se aprecia una escasa difusión en órganos oficiales de los contenidos referidos a temas fronterizos a través de la Difrol, ${ }^{5}$ pues su agenda prioriza el resguardo de las fronteras y límites por sobre el desarrollo de espacios transfronterizos.

Por ello se debe asumir su naturaleza ambigua y polivalente, y la validez de realizar sobre ella múltiples aproximaciones (Medina, 2006:12). Desde esta constatación, sobre todo es clave desentrañar las nuevas reivindicaciones identitarias y representaciones sobre el "otro", más allá de la frontera (Linares, 2009). En síntesis, las causas de estos nuevos fenómenos podemos indagarlas, primero, según si se trate de actores del desarrollo, que tienen una mirada particular hacia la posibilidad de profundizar el desarrollo transfronterizo, o de autoridades que deben velar por la soberanía, el patrimonio fitosanitario del país y el orden público, entre otros mandatos. Así, esta frontera, como muchas de América Latina, tendría dentro de sus características el encuentro conflictivo con el "otro". Por ello, estos territorios también se constituyen en "un espacio de disputa y tensión. Un espacio en donde surgen, entre otras cosas, discursos identitarios basados en la discriminación y la afirmación de la 'nacionalidad', justo cuando 'lo nacional' está dejando lugar a otro tipo de concepción económica-territorial" (Linares, 2009).

\section{La región de Tarapacá en la trama internacional subnacional y en el contexto bilateral Chile-Bolivia}

Desde mediados del siglo xix, Tarapacá ha sido una región con vocación internacional, producto de su carácter cosmopolita en torno al Ciclo del

\footnotetext{
${ }^{5}$ La Dirección Nacional de Fronteras y Límites del Estado (DIFROL) es un organismo técnico del Ministerio de Relaciones Exteriores de Chile, su misión es preservar y fortalecer la integridad territorial del país, asesorando técnicamente al gobierno y participando en la celebración de tratados, en la negociación de convenios, así como en los foros y materias relacionadas con los límites internacionales de Chile y las políticas de integración física, vecinal y regional (ver: http://www.difrol.cl/index.php?option=com_conte $n t \&$ task $=$ category\&sectionid $=8 \&$ id $=41 \&$ Itemid $=20)$.
} 
Salitre (Gavilán y Tapia, 2006; González, 2002). Su desarrollo ha dependido del entorno subregional y particularmente de sus vínculos comerciales con regiones bolivianas. Actualmente, Tarapacá se constituye como una región estratégica para el Centro-Oeste de Sudamérica, considerando que la condición de puente entre el mercado sur asiático y la subregión sudamericana dinamiza los intercambios presentes en los mercados locales de los países que conforman esta zona. Así, las relaciones comerciales entre Bolivia y Chile, a través de los puertos chilenos de Arica e Iquique, se han incrementado ostensiblemente en la última década (Sánchez, 2005).

En este proceso de larga data, también ha contribuido una serie de acercamientos sostenidos por Chile y Bolivia tras los acuerdos que sucedieron al fin de la guerra del Pacífico. Estas iniciativas han ofrecido un campo para acciones paradiplomáticas de actores presentes en estas fronteras: "A pesar de los cien años de un conflicto diplomático, en este plano de los acuerdos bilaterales ha habido avances, a saber: el tratado de 1904, las Convenciones sobre tránsito de 1912 y 1937, la Declaración de Arica de 1953, los acuerdos de Complementación económica de 1955" (González, 2006:115), adicionando el más reciente Acuerdo de Complementación Económica de 1994, junto a la vigente agenda de 13 puntos que contempla un "diálogo sin exclusiones" entre las autoridades de ambos países, incluyendo desde el tema marítimo hasta aspectos socioculturales.

A pesar estos avances en la relación vecinal, el escollo en torno de la mediterraneidad de Bolivia ha tenido un fuerte impacto en la proyección transfronteriza de la región de Tarapacá. ${ }^{6}$ En este sentido, los fundamentos de la política exterior chilena hacia Bolivia han seguido una política del statu quo en torno al tema marítimo, amparada en el legalismo y su expresión de adhesión a la "no revisión de los tratados". Las relaciones diplomáticas y los avances en la integración bilateral han estado cruzados por la existencia de puntos de vista antagónicos sobre el tema marítimo, donde la posición de Chile ha tendido a afirmar el carácter exclusivamente bilateral en torno a este tema, no aceptando el derecho de terceros a

\footnotetext{
${ }^{6}$ La pérdida del acceso al mar de Bolivia supone un costo que se interpreta en el imaginario colectivo boliviano como una desmembración cualitativa, la guerra del Pacífico pasó a representar la "guerra de las guerras y la cuna de la desconfianza hacia Chile (Francisco, 2009:47).
} 
intervenir, siguiendo una línea enmarcada dentro de la perspectiva realista de las relaciones internacionales (Francisco, 2009:57) que limita las opciones de integración dentro de la subregión.

Las limitantes políticas a la integración han transitado, sin embargo, en paralelo con la prioridad otorgada por Chile a la internacionalización económica. En la actualidad, a partir de la consolidación de la Zona Franca de Iquique (Zofri), la región de Tarapacá está mostrando especiales condiciones para constituirse en una plataforma de servicios, donde el establecimiento de un corredor bioceánico en la zona sería el producto más emblemático de la complementariedad económica en las áreas fronterizas. ${ }^{7}$ Los corredores de exportación a través de carreteras internacionales entre Bolivia, Chile y Brasil se han consolidado lentamente, al punto de que para finales de 2010 se inaugurará un corredor que vinculará a los tres países y unirá el Atlántico con el Pacífico, ${ }^{8}$ haciendo más compleja la red de interconexión transfronteriza.

Con todo, una preocupación más actual de la política exterior, paralela al desafío de lograr que la región de Tarapacá se convierta en el gran centro de distribución del comercio exterior boliviano, generando polos

\footnotetext{
${ }^{7}$ Así lo detalla categóricamente el presidente del directorio de la Sociedad Administradora de Zofri, Felipe Pérez Walker: "Las ventas de la Zona Franca de Iquique (Zofri) han venido creciendo sistemáticamente durante los últimos años, alcanzando el 2007 los 2.644 millones de dólares, un nuevo récord histórico. Este se verá largamente superado en 2008, esperándose llegar a los 3.300 millones de dólares. Esta cifra supera el total de las exportaciones agrícolas, frutícolas y ganaderas de Chile, es mayor a las exportaciones forestales, las de la industria del salmón, y equivale a su vez a más del doble de las exportaciones de vino. Zofri se ha transformado en la más importante zona franca comercial de Sud América, y en el principal y más gravitante centro de negocios del cono sur del continente. Su ubicación geográfica estratégica, constituye su principal ventaja competitiva, permitiéndole operar como un gran centro de distribución, principalmente para Bolivia, Perú y Paraguay [...] el corredor bioceánico le permitirá no sólo penetrar nuevos mercados, sino también concentrar una oferta exportable de proyecciones de países de la región que buscan exportar sus productos a través del Pacífico. Entre los mercados de destino de las mercancías de Zofri, Chile concentra el 53\% de ellas, seguido de Bolivia con un 26\%, Paraguay con un 9\% y Perú con un 8\%" (Pérez, 2008:3).

${ }^{8} \mathrm{Su}$ trayecto incluirá la Amazona brasileña, el altiplano boliviano y el norte chileno. El corredor unirá los puertos de Santos, Brasil (Atlántico) y Arica-Iquique, Chile (Pacífico), a través de Bolivia.
} 
de desarrollo, consiste en valorar la identidad regional de los actores involucrados en estas iniciativas. De ahí que exista un énfasis en el estímulo al desarrollo regional en zonas más deprimidas y abandonadas de ambos países, las cuales se verían favorecidas con la ampliación del comercio limítrofe, el fomento de zonas francas y el desarrollo de los servicios (Figueroa, 2009), como es el caso de la Alianza Estratégica Aymaras Sin Fronteras -caso que analizaremos más adelante.

En síntesis, la región estudiada sugiere una importante paradoja relacionada con la brecha existente entre el estímulo a la integración económica y la falta de mecanismos de sincronización política y vinculación sociocultural que dé cuenta de las dinámicas transfronterizas. La teoría de las RRII y su sometimiento a pruebas empíricas entrega interesantes elementos de análisis para entender esta contradicción y plantear las posibles soluciones a los dilemas de la integración.

\section{Explicaciones desde la teoría de las RRII}

El debate teórico presente entre las corrientes actuales del estudio de las políticas exteriores considera la discusión entre las posiciones racionalistas y las ubicadas dentro del paradigma reflexivo. Es decir, entre aquellas que ponen énfasis en los actores y el diseño de la política exterior, desde la óptica de la maximización de sus intereses por parte de grupos específicos, y aquellas que apuntan a la construcción de nuevas opciones compartidas en materia de políticas exteriores de interés común, en torno a las dinámicas presentes -para este caso- en las zonas transfronterizas.

Si bien los Ministerios de Relaciones Exteriores (Minrel) tradicionalmente ejercieron sus funciones teniendo en cuenta un escenario internacional anárquico, limitándose sus políticas a los ámbitos diplomáticos y militares, los cambios acaecidos en el contexto mundial-denotados por la globalización, una creciente interdependencia, incidencia de regímenes internacionales, diversificación e influencia de actores no estatales, entre otros elementos- han generado procesos de transnacionalización y han involucrado a múltiples unidades del Estado en los proceso de elaboración de la política exterior, además de expandir su ámbito de acción (Snyder, 1962; Viotti y Kauppi, 1999). 
Bajo este contexto, los Minrel han debido adaptarse a los cambios del entorno y diversificar sus agendas para hacer frente a las demandas que requiere un contexto internacional complejo y diferenciado. En este sentido, Hoffman (1991) apunta hacia el papel de las estructuras burocráticas y los procesos de toma de decisión como un componente esencial para la adaptación de los Estados a las restricciones y oportunidades que genera la interdependencia. Sin embargo, y sobre todo en los países menos desarrollados, dicha adaptación resulta dificultosa ya que los procesos de política exterior son colectivos e involucran una pluralidad de individuos, interactúan diversas agencias, se estructuran a partir de agendas, son más reactivos que anticipatorios y son percibidos como más o menos desorganizados y poco estructurados (Bacchus, 1974).

Es decir, el escenario actual plantea dos dificultades y adaptaciones importantes: la primera tiene que ver con la necesaria capacidad organizativa para responder eficientemente a la diversificación de la agenda internacional; y la segunda, con conciliar la diversidad de intereses que entran en juego a la hora de priorizar determinadas acciones de política exterior. Sobre este último punto, han surgido numerosas hipótesis para explicar las decisiones de los Estados en materia internacional. Por un lado, las visiones racionalistas sostienen que los actores buscan maximizar sus ganancias (Grieco, 1990; Grieco, Powell y Snidal, 1993) u obtener beneficios mutuos (Axelrod, 1984; Keohane, 1988). Por otro lado, las corrientes reflexivas apuntan al impacto de la subjetividad humana, a los valores, a las prácticas culturales comunes y a las ideologías subyacentes a las decisiones, entre otros elementos, que no necesariamente se derivan del cálculo racional de intereses y que constituyen las relaciones contemporáneas como un arreglo social (Keohane, 1988; Zacher y Matthew, 1995).

\section{Debate racionalista}

Las perspectivas racionalistas pueden dividirse a partir de dos líneas generales. La primera, denotada por el paradigma neorrealista, apunta a que los Estados buscan mejorar su posición en el sistema internacional respecto de sus competidores, obteniendo beneficios absolutos. La segunda, neoliberal, donde los Estados persiguen la cooperación como una estrategia para superar los problemas de confianza y las fallas de mercado, obtenien- 
do beneficios mutuos. A pesar de esta diferencia, tanto neorrealistas como institucionalistas neoliberales coinciden en que los países buscan aumentar su beneficio a través del sistema internacional.

El neorrealismo (Waltz, 1979) observa el Estado como un actor racional que busca la seguridad mediante los mecanismos que estime más convenientes para la preservación de sus intereses. Así, las alianzas o acuerdos de integración serían constituidos por los Estados como una forma de resguardar sus intereses nacionales mediante la obtención de determinados beneficios. Para esta perspectiva, las estrategias para maximizar los intereses nacionales se fundamentan en mantener la seguridad del Estado, satisfacer las demandas económicas de sectores políticamente relevantes y potenciar el prestigio internacional del Estado (Sanders, 2001:620-621). De esta manera, las decisiones sobre política exterior están denotadas por la posición del Estado en la estructura internacional y de los beneficios que pueda obtener de una situación de cooperación (Snidal, 1985). Sin embargo, el problema de la aproximación neorrealista es que no se explica el proceso de determinación del interés o el contexto histórico en que se enmarcan las decisiones de política (Sanders, 2001:624-625).

Por contrapartida, los aportes del liberalismo a la teoría de las RRII han problematizado la noción de Estado y le han conferido a los grupos que participan en él una mayor significancia (Panke y Risse, 2007), en orden a las determinadas definiciones de política exterior que se adopten. En este marco, el institucionalismo neoliberal, si bien comparte con el neorrealismo la visión de que los Estados buscan maximizar sus beneficios a través de la cooperación internacional, plantea una mayor autonomía de los actores por sobre los constreñimientos estructurales.

Para esta perspectiva, si las élites del Estado no prevén que la cooperación traerá beneficios para sus intereses, no se espera que surja la cooperación ni se establezcan instituciones que la faciliten; por el contrario, cuando los Estados se benefician de la cooperación, los gobiernos promueven instituciones que la facilitan (Keohane, 2001:678). Adicionalmente, los grupos o individuos que participan en las definiciones de políticas pueden, además de influir en el nivel interno, articular coaliciones y redes con sus contrapartes foráneas para incidir en los procesos internacionales (Keohane y Nye, 1988). 
Si bien los institucionalistas sostienen que auque la cooperación produce beneficios, ante la ausencia de un compromiso creíble por parte de los otros jugadores, cada actor prevé el peor de los escenarios, produciéndose resultados negativos (Keohane, 2001:679). Es decir, para esta perspectiva, la información que tengan los jugadores será determinante a la hora de cooperar, en desmedro de la importancia que asignan los neorrealistas a la estructura internacional para predecir el comportamiento de los actores.

En síntesis, las visiones “neo-neo” (ver Salomón, 2002) asignan una alta valoración al comportamiento racionalista de los actores internacionales a la hora de establecer acuerdos con otros Estados. Para los neorrealistas, primarán las relaciones estructurales de poder al momento de establecer dichos acuerdos; mientras que para los institucionalistas neoliberales dependerá de si los actores perciben que la cooperación traerá determinados beneficios. Para el caso estudiado, es posible observar ambas visiones en las definiciones de política exterior existentes a nivel central, donde primaría una visión racionalista en el establecimiento de acuerdos en el ámbito fronterizo, sin considerar, por ejemplo, los procesos y las dinámicas existentes en el ámbito subnacional que no se condicen necesariamente con una cooperación denotada por el interés racional desplegado por el Estado en el nivel central, sino que se identificarían con elementos vinculados al desarrollo de intereses e ideas comunes que coexisten en el nivel transfronterizo.

\section{El diseño racionalista de la política exterior chilena}

En Chile observamos el predominio de una visión racionalista, dominada por una agenda económica de inserción internacional que ha traído aparejada una adaptación burocrática del nivel central. Sin embargo, paralelamente, observamos una tendencia hacia la incorporación de nuevas temáticas vinculadas hacia las relaciones vecinales, que son incorporadas en el nivel intermedio y local de la administración del Estado, que no necesariamente pueden agruparse en torno al paradigma racionalista sino que más bien se ubican más hacia una aproximación reflexiva o constructivista. 


\section{El pragmatismo de la política exterior}

Con distintos énfasis y en atención a las circunstancias históricas, la política exterior chilena ha estado marcada por algunos elementos distintivos. Ente ellos, se ha reconocido la existencia de una tradición legalista y un estilo civil-pragmático en la conducción de los asuntos internacionales, lo cual implicaba una aceptación de las reglas del juego y de los centros de poder mundial (Wilhelmy, 1979; Muñoz, 1985). No obstante, el pragmatismo civil sería interrumpido por la inclusión de un nuevo estilo en política exterior durante el régimen militar (1973-1989), catalogado de "pretoriano-ideológico" y en el cual el autoritarismo burocrático militar incluiría mayores grados de centralismo en la gestión de la Cancillería y una visión marcada por el anticomunismo propio del periodo de la Guerra Fría (Muñoz y Tulchin, 1984), con aprehensiones hacia las fronteras contiguas en el marco de la lógica de la Seguridad Nacional (Atkins,1991).

A pesar de la irrupción de la dictadura militar, el retorno a la democracia (1990) propendería a un reposicionamiento de la tradición legal-pragmática, cuestión manifestada en el manejo de la política exterior llevada a cabo por parte de los gobiernos de la Concertación ${ }^{9}(1990-2010)$ y por la estructura institucional del Minrel. En efecto, como sostienen varios autores, la política exterior de Chile ha sido diseñada y conducida en atención a los cambios acaecidos en el contexto internacional, intentando adaptarse funcionalmente a los regímenes internacionales a través de la adscripción a las normas internacionales y de una estrategia de apertura que atañe a los ámbitos políticos, sociales, culturales y, particularmente, al aspecto económico (Rojas, 1997; Wilhelmy y Durán, 2003; Ross, 2006; Fuentes, 2007).

El gobierno de Patricio Aylwin (1990-1994) estuvo marcado por el fin de la dictadura militar y el consiguiente proceso de reinserción de Chile en el contexto internacional. En este periodo, el país vivió un proceso de adscripción a un entorno mundial caracterizado por el fin de la Guerra Fría y un contexto propicio para la incorporación a las instancias multilaterales

\footnotetext{
${ }^{9}$ Coalición de centro-izquierda formada a finales de la dictadura y gobernante en Chile entre los años 1990 y 2010, constituida por los partidos Demócrata Cristiano (PDC), Radical Socialdemócrata (PRSD), Por la Democracia (PPD) y Socialista (PS),
} 
y regímenes internacionales, desde el ámbito económico y financiero, hasta los mecanismos de coordinación política a nivel regional y mundial.

Si bien es cierto que los esfuerzos de reinserción internacional fueron evidentes, también es necesario advertir algunas carencias, propias de un periodo de transición a la democracia en el que las prerrogativas militares se mantenían con un alto nivel de impacto e influencia en las decisiones de política exterior, atravesadas, además, por una falta de coordinación y articulación en el nivel burocrático, donde se advirtieron déficits de coordinación dentro de la Cancillería y con otros sectores gubernamentales (Wilhelmy y Durán, 2003). A pesar de ello, el gobierno de Aylwin retomaría la tradición pragmática-legalista de Chile, cuestión característica de su política exterior histórica (Colacrai y Lorenzini, 2005).

Este reimpulso pragmático se evidenciaría y consolidaría durante la administración de Eduardo Frei (1994-2000), donde la activa participación internacional se sustentó en un modelo de desarrollo económico basado en el libre comercio y la apertura financiera. Tal como sostiene el ex canciller José Miguel Insulza, “un país abierto al mundo como el nuestro debe vincular necesariamente los intereses de su inserción internacional a sus relaciones exteriores. La dimensión económica de la política exterior adquiere así un carácter prioritario” (Insulza, 1998:18). En términos organizativos, los esfuerzos de la Cancillería se concentrarían efectivamente en modernizar su aparato burocrático a objeto de superar los déficits advertidos durante el gobierno de Aylwin, priorizando casi exclusivamente el aspecto económico de la internacionalización.

La situación anterior se materializaría durante el gobierno de Ricardo Lagos (2000-2006) a través de un fuerte impulso a la Dirección General de Relaciones Económicas Internacionales (Direcon), que vería incrementada su dotación de 98 a 281 funcionarios entre los años 2000 y 2005, funcionarios con un alto perfil técnico, provenientes de las áreas de la economía y del derecho (Fuentes, 2007:106). Estos ejemplos son un resultado de la prioridad por la apertura económica de Chile, plasmada además en la firma de una serie de tratados de libre comercio en la participación en foros internacionales como la APEC, entre otros, que forzaron a un crecimiento sostenido de las reparticiones públicas vinculadas a la materia.

Aunque durante estas administraciones también se procuró una mayor actividad en materias internacionales de corte más político, reflejado -por 
ejemplo- en la participación de Chile como integrante no permanente del Consejo de Seguridad o como miembro del Consejo de Derechos Humanos de Naciones Unidas, durante los gobiernos de Lagos y de Michelle Bachelet (2006-2010), no se advirtieron adaptaciones o cambios a nivel administrativo en la Cancillería, manteniendo la estructura tradicional del Minrel.

Si bien ha generado una adaptación de su política exterior, correspondiente con los cambios advertidos en el escenario internacional, el funcionamiento interno de chile ha tendido a ser incompleto e informal (Fuentes, 2007). El Minrel de Chile ha sido una de las pocas instituciones que no ha llevado a cabo un proceso de modernización institucional general que involucre a la totalidad de sus componentes. Pese a esto, la política exterior del país ha sido altamente abierta y adaptativa a los cambios del contexto internacional. Una de las explicaciones en este sentido, es que se ha desarrollado

[...] una burocracia paralela que complementa y soluciona las demandas fuera de un esquema burocrático tradicional. La adaptación a un marco de interdependencia compleja se ha dado a partir no de transformaciones organizacionales significativas, sino más bien mediante la institucionalización de mecanismos informales que ayudan a definir el curso de acción política al más alto nivel, con espacios flexibles de interrelación entre niveles medios y altos del aparato del Estado. Asimismo, tiende a generarse una burocracia paralela, altamente profesional, que responde a las demandas urgentes de la autoridad política (Fuentes, 2007:1).

La cita anterior denota la necesidad de conciliar el papel tradicional de los Minrel con agendas crecientes de política exterior, que para el caso chileno se ha producido, en cierta medida, de una forma no institucionalizada a través de la Direcon y de Pro Chile. ${ }^{10}$ En lo atingente a las regiones, el proceso de adaptación ha sido menor a través de la Dirección de Asuntos

\footnotetext{
${ }^{10}$ Instituciones que poseen mayor autonomía y flexibilidad respecto del Minrel y que han logrado importantes objetivos frente a los actores económicos del país y del exterior.
} 
Regionales ${ }^{11}$ (Dicore), organismo que no posee una estructura burocrática ni representantes oficiales en las regiones del país.

Si en el área prioritaria -económica- de inserción del país existe una falta de institucionalización de los procesos de política exterior en el nivel central, en aquellos sectores como las relaciones transfronterizas, la adaptación organizativa ha sido significativamente menor y por ende ha estado circunscrita a las agencias locales o vinculadas al desarrollo regional, generándose una falta de vinculación de éstos con los agentes de decisión de política exterior.

Por ejemplo, el diagnóstico que hace la agrupación de empresarios y gobiernos regionales de regiones fronterizas que agrupa a las zonas del noroeste argentino, norte de Chile y centro-este de Bolivia, Zicosur (Zona de Integración del Cono Sur de Sudamérica), ${ }^{12}$ es lapidario. Pese a ser una de las zonas más integradas en que se involucran actores subnacionales, "la vinculación con los organismos nacionales que tienen injerencia en la generación y ejecución de la política internacional de Chile es deficitaria, sólo en casos coyunturales se reciben apoyos a través de la Dirección de Fronteras y Límites (Difrol)" ${ }^{13}$ Existen otras organizaciones de la institucionalidad pública y privada que adelantan proyectos integracionistas propios que en ciertos casos coinciden y en otros son diametralmente opuestos a los de los gobiernos regionales, como ocurre en foros de integración o en determinadas municipalidades, por mencionar algunos ejemplos.

La adaptación al contexto internacional ha seguido un camino centrado en aquellos aspectos económicos que coinciden y profundizan la tradición de política exterior legal-pragmática. En términos teóricos, el diseño de la política, sumado a una falta de modernización de las estructuras administrativas, obedece esencialmente a patrones racionalistas, dada la

${ }^{11}$ La Dirección de Coordinación Regional es responsable de apoyar y coordinar a las regiones y municipios del Chile en la realización de sus proyectos e iniciativas en el ámbito internacional. Para ello, la DICORE permite vincular a los gobiernos regionales y locales con las direcciones del Ministerio de Relaciones Exteriores y la red de embajadas, misiones y consulados Ver: http://www.minrel.gov.cl/prontus_minrel/site/ artic/20080903/pags/200809031113 14.php\#T0).

${ }^{12}$ Ver: http://www.zicosur.com/www/php/que_es.php

${ }^{13}$ En www.minrel.gov.cl/.../n_541_experiencia_regional_en_cooperacion_transfronteriza.ppt 
primacía otorgada a la apertura económica que ha sido acompañada de cambios $a d-h o c$ a esta forma de direccionar las relaciones del país. El escaso énfasis en cuestiones vinculadas a temáticas fronterizas no tradicionales ${ }^{14}$ -caracterizadas por un creciente dinamismo e intercambio- denotaría una falta de preocupación por esta emergente área de las relaciones entre países como consecuencia de los pocos beneficios que representaría para el poder central su profundización, en comparación con tratados de libre comercio o foros internacionales tendientes a la generación de diversos acuerdos comerciales.

Como se ha señalado, tanto a escala internacional, a través de mecanismos de integración, como a escala nacional, a través de la Cancillería, la promoción del desarrollo fronterizo o transfronterizo es muy débil, dado que se focaliza más bien en las políticas macroeconómicas y macroterritoriales. Las razones apuntarían a la preeminencia de una racionalidad tecnocrática que ha liderado el proyecto de internacionalización de la economía chilena en las últimas décadas, lo que los ha posicionado como actores claves en la implementación de políticas y programas (Silva, 2006), a los que no escapa la política exterior.

\section{Debate reflexivo}

En términos generales, el constructivismo apunta a la idea de construcción social, mediante un proceso de interacción de identidades que van moldeando las prácticas internacionales nuevas, ocurridas luego del "corrimiento de velo" tras el fin de la Guerra Fría (Russell, 1996), y que ocultaba el real significado de estas transformaciones.

Las respuestas que aporta el renovado debate dentro de las RRII, donde se inscribe esta corriente, apuntan al "vínculo entre conocimiento, historia, cultura y relaciones de poder y prestan más atención al lenguaje, a la construcción lingüística y al discurso" (Vieira, 2005:272-273). Desde esta perspectiva, el constructivismo busca redefinir nuevos intereses e identidades e institucionalizar nuevas relaciones sociales en el campo de las RRII y particularmente en los procesos de integración a distinta escala.

\footnotetext{
${ }^{14}$ Entendiendo por 'tradicionales' aquellos temas vinculados a la defensa, seguridad y protección del territorio soberano.
} 
Los procesos de cambio pacífico en las RRII contemporáneas y su dinámica cooperativa, por sobre la estructura existente, ha sido una prioridad de las aproximaciones constructivistas (Wendt, 1999, 2005; Salomón, 2002) Así, pueden reflejar la trama compleja de relaciones generadas por la interacción de nuevos actores de la arena internacional -gobiernos locales y regionales, sociedad civil e instituciones del sector privado-. Desde esta perspectiva, se presta énfasis al análisis de las identidades de los actores y no sólo a las conductas y decisiones racionales de éstos.

La construcción de nuevas identidades, resultado del surgimiento de nuevos espacios emergidos de las integraciones periféricas transfronterizas, sería uno de sus nuevos objetos de estudio. En efecto, el tratamiento de los factores sociocognitivos (Salomón 2002:37) se apreciaría al concentrarnos en los actores locales que interactúan en el plano fronterizo, pues las relaciones que ahí se suscitan no corresponderían necesariamente a una cooperación orientada por el interés racional, sino que se identificarían elementos vinculados al desarrollo de intereses e ideas comunes que surgen del nuevo aprendizaje en torno al "municipalismo y la cooperación descentralizada”. Este argumento se ve reforzado en la medida en que el aumento de las capacidades internacionales para gestionar la acción exterior de actores subnacionales estaría validando estas nuevas escalas de acción internacional transfronteriza a través de su "institucionalización social, material y espacio-temporal" (Jessop, 2004). Se trata de enfatizar que siempre será preferible que los acuerdos estratégicos sean sancionados o socializados con las comunidades o sociedades reales, para darle no sólo legitimidad social sino proyección de largo plazo (González, 2006). Es decir, se trata de reconocer el nuevo acontecer internacional subestatal, caracterizado por una falta de institucionalización de sus prácticas pero signado por la emergencia de un decidido discurso municipalista, sobre todo en actores claves del desarrollo fronterizo de carácter étnico.

En consideración de estos antecedentes, el constructivismo recoge la forma en que las prácticas cognoscibles constituyen sujetos -surgida de la socialización y el aprendizaje mutuo-, por tanto suponen un concepto intersubjetivo del proceso en el que las identidades y los intereses son endógenos a la interacción, en lugar de ser exógenos y dados, como apunta el concepto racionalista representado por las corrientes neo-neo (Salomón, 2002). En la medida en que "los actores tienen múltiples identidades, y 
que estas identidades implican intereses diferentes, la atribución exógena y a priori de intereses idénticos a todos los Estados es inválida” (Hopf, 1998:199). Así, si reconocemos que el Estado no es un actor monolítico y se descompone espacialmente en distintas escalas de acción -local, regional, nacional, internacional, interméstica- con distintos intereses y prioridades, es plausible argumentar que estas nuevas escalas de acción transfronteriza estarían construyendo nuevas identidades. En efecto, los procesos de integración transfronteriza requieren de una transformación simultánea en diferentes niveles de actividades sociales relevantes para el potencial de integración. Además de interacción económica a través de la frontera, interacción de actividades políticas a diferentes niveles gubernamentales, en específicas influencias políticas de comunidades adjuntas, y en sus culturas (Eskelinen, 2010).

No obstante, esta construcción no estaría exenta de conflictos, toda vez que estos proyectos locales socavan las soberanías nacionales y sus formas de control territorial, sobre todo si se trata de Estados centralistas y verticalistas como es el caso latinoamericano; incurriendo éstos en estrategias que usualmente limitan despliegue e institucionalización de los agentes subnacionales (Tapia, 2003). Además, es aún más complejo cuando ya no se trata sólo de cooperación internacional, sino de cooperación descentralizada; es decir, de procesos de colaboración que legitiman nuevas escalas de acción que el Estado nacional no siempre controla o legitima, teniendo en cuenta ciertos elementos culturales ${ }^{15}$ y clivajes que de alguna manera socavan las opciones integracionistas.

Con todo, si bien esta perspectiva plantea la posibilidad de construir nuevas realidades en el ámbito internacional, que sean objeto de políticas, estas últimas, sobre todo las emanadas desde el poder central en Chile, poseen características más centradas en elementos racionales que reflexivos, puesto que la institucionalización de estos espacios no ha sido una

\footnotetext{
${ }^{15}$ Así por ejemplo, Jean Der Derian plantea la idea de ciertas culturas diplomáticas determinantes en el derrotero de las políticas exteriores de los países. Para este autor, la genealogía es una manera apropiada de desentrañar los significados de una cultura diplomática, pues ésta pone en tela de juicio los orígenes inmaculados, las identidades esenciales y las estructuras profundas de la teoría internacional (Der Derian, 1987), sobre todo sus dogmas realistas como es el interés nacional, la conducción central de las relaciones exteriores, la unidad de la diplomacia y la política exterior, entre otras.
} 
prioridad en la agenda exterior. Sin embargo, como se ha sostenido, en las relaciones transfronterizas observamos una creciente vinculación a partir de las experiencias subnacionales, que sugiere una aproximación más centrada en elementos constructivistas.

En efecto, el surgimiento de referenciales no tradicionales, en el caso de la política exterior chilena hacia las fronteras, a partir del discurso municipalista y el que promueve la cooperación descentralizada, plantea el tema de la pertinencia de nuevos actores en la formulación e implementación de dicha política, tanto en las instancias de discusión -foros- como en las disputas entre ellos por el poder -arenas- (Jobert, 2004).

\section{La experiencia subnacional: Proyecto Estratégico Aymaras Sin Fronteras}

Un actor clave en este nuevo escenario subnacional es la Asociación de Municipios Rurales de la Región de Tarapacá ${ }^{16}$ que, junto a sus pares bolivianos -municipios de La Paz, Oruro- y peruanos -Tacna, Puno-, emprendieron el proyecto Alianza Estratégica Aymaras Sin Fronteras. Ésta es una organización internacional sin fines de lucro, de carácter intermunicipal, creada el año 2001, que mediante la articulación y cooperación a través de las fronteras busca implementar políticas que fomenten el desarrollo de la comunidad en áreas como agricultura, infraestructura, pequeñas empresas, recursos naturales, energía, cultura, educación, salud y desarrollo económico.

Francisco Humire, alcalde de Putre y primer presidente de esta asociación, ha sostenido que se ha aceptado la institucionalidad vigente, caracterizada por la articulación de actores a distinta escala, para el logro de lo objetivos de la zona y para poder tener acceso a la escena internacional con apoyo técnico gubernamental y no gubernamental:

Si bien hemos logrado que los organismos de cooperación internacional se fijen en la Alianza, y por otro lado los organismos como la Cancillería también

\footnotetext{
${ }^{16}$ El organismo posee una vasta experiencia internacional a partir de vivencias de integración transfronteriza entre municipios rurales de Chile y Bolivia, (ver: http://www. cooperaciondescentralizada.gov.cl/1511/article-69494.html).
} 
se preocupen de nuestra iniciativas, es imposible pensar la gestión de la Alianza como esfuerzos aislados [...] Los apoyos de los Gobiernos Regionales son importantes, ya que muchas de las aplicaciones de los intereses de la Alianza pasan por la sensibilidad que ellos y otros agentes regionales tengan hacia el mundo Aymara. ${ }^{17}$

No obstante, Humire considera que por su sustento cultural, los municipios poseen mayor claridad acerca del desarrollo transfronterizo que las autoridades regionales, aunque entiende que su colaboración es relevante, sin perder con ello su finalidad última: la unidad aymara trinacional, dando cuenta del enfoque territorial que demandan los proyectos de cooperación transfronteriza. En palabras del propio Humire, lo destacable de la Alianza es precisamente su carácter étnico:

Nosotros tenemos un sustento cultural que nos hace distintos a otras regiones ya que somos Aymaras. La Alianza busca otorgar fuerza al movimiento indígena haciendo resurgir una organización que tiene un pasado milenario. Desde tiempos de Tiwanaku que esta zona tiene algo especial, una concepción regional con una base étnica que es necesario reivindicar. ${ }^{18}$

En efecto, el creciente auge del municipalismo transfronterizo demanda nuevas estructuras territoriales y exige asumir proyectos socioculturales que combinen identidades heredadas con aperturas a la diversidad (Alburquerque, 2004). Siguiendo esta argumentación, observamos que la perspectiva de Humire no se contradice con el poder político de los Estados nacionales, sino más bien es una demanda institucional, donde lo político se pone al servicio del requerimiento étnico (González et al., 2008):

No descuidamos el pasado y nuestras reivindicaciones como pueblo, estamos muy conscientes que a nivel comunal y a nivel territorial, cada municipio y cada alcalde tiene y enfrenta problemas concretos que tienen que ver con la pobreza y marginalidad. Si nosotros como autoridades con ascendencia e identidad indígena Aymara no somos capaces de atacar y solucionar estos

${ }^{17}$ Entrevista realizada por el autor a Francisco Humire, febrero de 2008.

${ }^{18}$ Entrevista realizada por el autor (González et al., 2008). 
problemas, es difícil que otra persona sin esta visión regional lo haga [...] Nuestra labor de Alcaldes nos hace -por la naturaleza de nuestro cargo- tener una visión regional y tener conciencia plena de los problemas de nuestras comunidades. Ahora no es novedad que las autoridades rurales tengan la noción de macro-región, entonces si las particularidades de la zona Aymara son las mismas en los tres países, el sentido común nos lleva a buscar soluciones comunes para la región Aymara. ${ }^{19}$

Tomando en cuenta el itinerario del proyecto Aymaras Sin Fronteras, otro actor clave es la Subdere, fundamentalmente por su papel articulador entre los actores locales y nacionales involucrados en la emergencia de proyectos transfronterizos, papel articulador demandado desde el gobierno central. En este sentido, un elemento importante a tener en cuenta apunta a que se trata de la intervención en una zona fronteriza percibida como compleja y estratégica; elementos propios de un territorio que posee condiciones estructurales históricas de cierta conflictividad latente, permeada por elementos de política exterior de corte pretoriano-ideológico. Por ello, se requería sobre todo en Aymaras Sin Fronteras la venia de los respectivos Estados nacionales para consolidar dicho proyecto.

Desde esta perspectiva, si se sigue el itinerario de dicho proyecto, queda en evidencia la aprensión del Estado central hacia esta iniciativa innovadora. En efecto, a partir de la escasez de fondos públicos para su implementación, la iniciativa se estanca en 2006, oportunidad para que intervengan los Estados, aunque esta intervención se realiza de manera tardía, pues la iniciativa ya llevaba cuatro años de funcionamiento. En este contexto, el Estado chileno gestionó un proyecto de inversión vía cooperación internacional a través del Banco Interamericano de Desarrollo.

Paradójicamente este tipo de fuentes de financiamiento considera como fundamentales las iniciativas que promueven nuevas formas de gobernanza a partir del municipalismo y la cooperación descentralizada (Rouviere, 2009). De ahí que se gestionara el proyecto mediante un funcionario de la Subdere instalado en el GORE. Efectivamente, el encargado de liderar el proyecto, Helmut Häffelin, señalaba: "para el BID el proyecto tiene mucha más identidad si lo presenta una entidad regional que una central". ${ }^{20}$ Es

${ }^{19}$ Entrevista realizada por el autor (González et al., 2008).

${ }^{20}$ Para conocer la entrevista en profundidad, ver Rouviere (2009:17). 
decir, se presentaba al BID un proyecto desde lo regional, aunque mediante un funcionario proveniente del nivel central, generando una organización ad-hoc no institucionalizada.

En este mismo sentido, un punto que llama la atención es que esta trama de actores que operan a distintos niveles evidencia más conflictos por espacios de poder entre distintos niveles de gobierno e instituciones, dentro de cada país, que entre homólogos municipales o regionales en los tres países (Rouviere, 2009:19), situación que evidencia una incipiente institucionalidad y la falta de adaptación de la política exterior hacia las dinámicas fronterizas.

\section{Conclusiones}

La construcción de la actual frontera del norte de Chile, al igual que todos los territorios fronterizos en transición que han experimentado procesos de largo repliegue e incipiente apertura, ${ }^{21}$ apunta a la existencia de controles fronterizos y un interés por la integridad territorial de manera que la división entre actores que están dentro y fuera de la comunidad -"nosotros" y "ellos"- están en principio bien definidos (Eskelinen, 2010). Aunque, simultáneamente, la política de desarrollo regional-local y las actividades de ordenación del territorio estarían inspirándose en nuevos procesos de apertura y gobernanza a distintos niveles.

En el caso analizado, las políticas públicas de sello territorial muestran tendencias hacia una identidad étnica que tiene alcances transfronterizos visibles $^{22}$ que requieren de una transformación simultánea en diferentes

\footnotetext{
${ }^{21}$ Nos referimos a procesos de larga duración como han sido los efectos de guerras fronterizas, presencia de doctrinas militares obsesionadas con el control de las zonas contiguas, presencia de dictaduras militares que apelan al nacionalismo, entre otros elementos de carácter histórico-cultural y también coyuntural (ver Milet, 2004; Álvarez y Fuentes, 2010).

${ }^{22}$ Así se evidencia en las conclusiones planteadas en el Seminario de Integración Fronteriza de la Triple Frontera (Bolivia, Chile y Perú), realizado el 9, 10 y 11 de marzo del 2008 en la ciudad de Tacna, Perú. Dentro del acta final se consideró que los requerimientos técnicos y/o políticos para todas las políticas públicas que se diseñen para la región tri-fronteriza, como para todo proyecto de desarrollo de agencia estatal, privada, nacional o internacional, debe considerar a la cosmovisión andina como parte esencial, con la finalidad de una mejor pertinencia y eficacia de esas políticas y proyectos.
} 
campos de actividades sociales relevantes para el potencial de integración, por ejemplo, a partir de la presencia de un régimen transfronterizo más o menos institucionalizado.

Cabría preguntarse entonces si los procesos presentes en la región apuntarían a la construcción de un régimen fronterizo institucionalizado. $\mathrm{O}$, de otra forma, ¿estamos en presencia de procesos de mayor complejidad a la usanza de la denominada "gobernanza multinivel?" ${ }^{23}$ La respuesta es negativa, de lo expuesto sólo se avizora un muy escaso desarrollo en la iniciativa de Aymaras Sin Frontera.

Además de la interacción económica a través de la frontera, no se advierte una interacción política en los distintos niveles gubernamentales que incluya la complejidad de las dinámicas transfronterizas. Mientras las actividades comerciales y de desarrollo están provocando incipientes cambios en las estrategias, regulaciones e iniciativas concernientes al régimen fronterizo, junto con la emergencia de nuevas identidades, todavía hay cuestiones pendientes para consolidar dicho régimen a un nivel políticogubernamental.

Entre ellas: escaso soporte y control de las iniciativas por parte de los centros; falta de descentralización y vinculación de la política exterior con las zonas fronterizas; además de la dualidad de funciones entre algunos actores por falta de un marco institucional. Este escenario, adicionalmente, redunda en la falta de apoyo de los organismos de financiamiento internacional -ya que éste está supeditado a una mayor profundización de la descentralización y la emergencia de nuevas formas de gobernanza-.

En cuanto al debate sobre política exterior reflexiva o racional, los nuevos referenciales han tendido a articular nuevas demandas en el ámbito de la política exterior, lo que se refleja, por ejemplo, en los comités de frontera. No obstante, su falta de institucionalización y resultados erráticos dejan a este nuevo discurso en una situación marginal, manteniéndose una política más centrada en aquellos intereses inmediatos derivados

${ }^{23}$ Lo central de esta estrategia apunta a la trama de actores que participan indistintamente en escalas estatales, supraestatales y subestatales, disputando competencias a los Estados-nación y con implicaciones políticas en la construcción de las identidades territoriales emanadas de estas instituciones que interactúan en distintos ámbitos relacionados (Marks, 1993:392). 
del cálculo económico, que de opciones de integración y cooperación que recojan las especificidades de la región transfronteriza. Esta situación es coadyuvada por las tradiciones presentes en la política exterior chilena y por una falta de desarrollo organizativo y de modernización del Minrel.

En los hechos, a pesar de que Aymaras Sin Fronteras se ha articulado en instancias de gobernanza multinivel, a seis años de su funcionamiento, se evidencia que una de las características de esta nueva estructura en materia internacional subnacional es su baja institucionalización y escaso soporte desde el nivel central.

Una nueva pauta regularizada de interacción entre los actores locales, nacionales e internacionales, conocida, practicada y aceptada, pero no necesariamente aprobada por todos (O’Donell, 1996), tiene expectativas relativas de guiar eficazmente la gestión internacional de acuerdo con las reglas sancionadas y sostenidas por ella. Sin embargo, la persistencia de elementos tradicionales en las definiciones de política exterior, acompañados de una racionalidad económica en su accionar, no sugiere en el corto plazo un cambio en las relaciones vecinales. Tal como plantea el debate racionalista, si desde el poder central no se prevé que la cooperación traerá beneficios, no se espera que surja la cooperación ni se establezcan instituciones que la faciliten, cuestión que se vería reforzada por el deterioro de las relaciones chileno-bolivianas en el último tiempo, sumados a los problemas internos que enfrenta cada país.

Bajo esta lógica, considerando la omisión de las particularidades fronterizas en la política exterior, se tenderá al mantenimiento del statu quo, privilegiando los temas tradicionales -inclusive con la posibilidad de agudizar los conflictos existentes-, al costo de limitar las probabilidades de integración y cooperación a partir de procesos de intercambio intersubjetivo para la (re)definición de los intereses (inter)estatales.

\section{Bibliografía}

Axelrod, Robert (1984), The Evolution of Cooperation, Basic Books, Nueva York. Alburquerque, Francisco (2004), "Desarrollo económico local y descentralización en América Latina", Revista de la CEPAL, 82: 157-171.

Álvarez, Gonzalo y Claudio Fuentes (2010), "Chile-Perú: un amargo pisco sour", Análisis del Año 2009, FACSO-Universidad de Chile, Santiago. 
Aranda, Gilberto, Cristian Ovando y Alejandro Corder (2010), "Experiencias paradiplomáticas en la región de Tarapacá y su proyección subregional”, Estudios Internacionales, 165:33-74

Atkins, Pope (1991), América Latina en el sistema político internacional, GEL, Buenos Aires.

Bacchus, William (1974), "Diplomacy for the "70s: An Afterview and Appraisal", The American Political Science Review, 68(2): 736-748.

Botero-Ospina, María Helena y Luis Cardozo (2009), "La regionalización de las relaciones internacionales en un Estado centralista: análisis del caso colombiano a partir de la Constitución de 1991", Papel Político 2(14): 609644.

Colacrai, Miryam y María Lorenzini Elena (2005), "La política exterior de Chile: excepcionalidad o continuidad? Una lectura combinada de "fuerzas profundas" y tendencias", Confines, 1-2: 45-63.

Debuyst, Fréderic (2009), "Lógicas y sentidos de los enfoques territoriales", Revista Polis, 8(22): 21-37.

Der Derian, James (1987), On diplomacy, Blackwell, Nueva York.

Eskelinen, Heikki (2010), "Formas volátiles y sostenibles de interacción como conductores de cambio en regiones fronterizas en el norte europeo”, Si Somos Americanos. Revista de Estudios Transfronterizos, 11(2):

Figueroa, Gonzalo (2009), "Chile y Bolivia en el MERCosur: Análisis y perspectivas", Diplomacia, 118: 70-124.

Francisco, Mila (2009), "La cuestión marítima en la política exterior de Chile y Bolivia”, Diplomacia, 118: 47-69.

Fuentes, Claudio (2007), “Internacionalización sin «modernización»: el caso del Ministerio de Relaciones Exteriores de Chile”, América Latina Hoy, 46: 97117.

Gallardo,Abel(2007), "La dimensión subnacional de las relaciones internacionales", Diplomacia, 110: 40-58

Gavilán, Vivian y Marcela Tapia (2006), "Diagnóstico de los procesos migratorios en el norte de Chile", Revista Electrónica Parinas, 2(1).

González, Sergio, Laetitia Rouviere y Cristian Ovando (2008), “De 'Aymaras en la Frontera'” a '“Aymaras Sin Fronteras"': Los gobiernos locales de la triplefrontera andina (Perú, Bolivia y Chile) y la globalización, Revista Diálogo Andino, 31: 31-46.

González, Sergio (2006), "Densidad, integración y conflicto en la triple frontera (Perú, Bolivia, Chile)", Serie integración social y fronteras, Convenio Andrés Bello, Bogotá.

González, Sergio (2002), Hombres y mujeres de la pampa. Tarapacá en el ciclo de expansión del salitre, LOM Ediciones, Santiago. 
Grieco, Joseph (1990), Cooperation Among Nations: Europe, America, and Non-Tariff Barriers toTrade, Cornell University Press, Ithaca.

Grieco, Joseph, Robert y Powell Duncan Snidal (1993), “The Relative-Gains Problem for International Cooperation”, The American Political Science Review, 87 (3): 727-743.

Hoffmann, Stanley (1991), Jano y Minerva. Ensayos sobre la Guerra y la Paz, GEL, Buenos Aires.

Hopf, Ted (1998), "The promise of constructivism in International Relations theory", International Security, 23 (1): 171-200.

Insulza, José Miguel (1998), Ensayos sobre política exterior de Chile, Los Andes, Santiago.

Jessop, Bob (2004), "La economía política de la escala y la construcción de las regiones transfronterizas", EURE 29 (89): 25-41.

Jobert, Bruno (2004), Estado, sociedad, políticas públicas, Lom, Santiago.

Keohane, Robert (2001), "Relaciones Internacionales: lo viejo y lo nuevo", en R. y Goodin H. Klingemann (eds.), Nuevo manual de Ciencia Política, tomo II, Istmo, Madrid, pp. 668-692.

Keohane, Robert (1988), Después de la hegemonía, GEL, Buenos Aires.

Keohane, Robert y Joseph Nye (1988), Poder e interdependencia. La política mundial en transición, GEL, Buenos Aires.

Linares, María Dolores (2009), “Un puente en la zona transfronteriza: representaciones sociales, identidades y conflicto. El caso PosadasEncarnación”, Estudios fronterizos, 10(20): 47-77.

Marks, Gary (1993), "Structural Policy and Multinivel Governance in the EC", en Cafruny (ed.), The State of the European Community, Harolw, Boulder.

Medina, Eusebio (2006), "Aportaciones para una epistemología de los estudios sobre fronteras internacionales”, Estudios Fronterizos, 17(13): 9-27.

Milet, Paz (2004), "Chile-Perú: las dos caras de un espejo", Revista de Ciencia Política, 24(2): 228-235.

Meza Monge, Nilo (2005), “Zonas de integración fronteriza. Peru-Bolivia, una experiencia a considerar”, Aldea Mundo, 10(19): 36-43.

Moncayo, Edgar (2002), "Nuevos enfoques teóricos, evolución de las políticas regionales e impacto territorial de la globalización”, Serie Gestión Pública, ILPEs, 27: 51-65.

Muñoz, Heraldo (1985), “La política exterior de Chile: la crisis continúa”, en Heraldo Muñoz (comp.), Anuario de políticas exteriores latinoamericanas. Las políticas exteriores latinoamericanas frente a las crisis, GEL-PROSPEL-CERC, Buenos Aires.

Muñoz, Heraldo y Joseph Tulchin (1984), Entre la autonomía y la subordinación: Política exterior de los países latinoamericanos, GEL, Buenos Aires. 
O’Donnell, Guillermo (1996), “Otra institucionalización”, Revista Política y Gobierno, 2: 219-244.

Panke, Diana y Thomas Risse (2007), “Liberalism”, en T. Dunne et al., International Relations Theory: Discipline and Diversity, Oxford University Press, Oxford.

Pérez Walker, Felipe (2008), “zOFri: el portaviones de Asia Pacífico para llegar a Sudamérica”, Boletín Asia Pacífico, 4-8.

Podestá, Juan (2003), La invención de Tarapacá: Estado y desarrollo regional en Chile, Ediciones Campvs, Iquique.

Rojas, Francisco (1997), "Chile: cambio político e inserción internacional, 19641997”, Estudios Internacionales, 120: 376-406.

Ross, César (2006), "Chile: los desafíos de la política exterior de Michelle Bachelet" Foreign Affairs En Español, abril-junio.

Rouviere, Laetitia (2009), “¿Un territorio político transfronterizo? Formas de legitimación de una experiencia de legitimación de una experiencia de acción política intermunicipal”, Si Somos Americanos. Revista de Estudios Transfronterizos, 9(2): 13-29

Russell, Roberto (1996), "Cambios y continuidades en las relaciones internacionales”, en Capítulos del SELA, 47: 17-35.

Salomón, Mónica (2002), "La teoría de las relaciones internacionales en los albores del siglo xxi: Diálogo, disidencia, aproximaciones”, СIDOв d'Afers Internacionals $s, 4: 7-52$

Sánchez, Rigoberto (2005), Chile y Bolivia: Intereses comunes. La oferta portuaria de la región de Tarapacá al servicio del comercio exterior de Bolivia, Instituto de Estudios Internacionales, Universidad Arturo Prat, Iquique.

Sanders, David (2001), "Relaciones Internacionales: neorrealismo y neoliberalismo”, en R. Goodin H. Klingemann (eds.), Nuevo manual de Ciencia Política, tomo II, Istmo, Madrid, pp. 619-644.

Santos, Antonia (2010), "reflexiones en torno a los procesos de descentralización política en las regiones fronterizas del norte de Chile”, ponencia presentada en simposio Migraciones y Espacio Transfronterizo desde enfoques interdisciplinarios, Universidad de Santiago de Chile, Santiago, 29 de octubre al 1 de noviembre.

Silva, Patricio (2006), "Los tecnócratas y la política en Chile: pasado y presente", Revista Ciencia Política, vol. 26, núm. 2, 175-190.

Snidal, Duncan (1985), "The Limits of Hegemonic Stability Theory", International Organization, 39(4): 579-614.

Snyder, Richard (1962), Foreign Policy Decision-Making, Free Press, Nueva York.

SUBDERE (2009), Cuadros descriptivos de la actividad internacional de las regiones de Chile, Unidad de Relaciones Internacionales de la SubDERE, Ministerio del Interior de Chile, Santiago. 
Subdere (2008), Actualidad de las relaciones paradiplomáticas y de Ccooperación descentralizada de los municipios chilenos, Unidad de Relaciones Internacionales de la subdere, Ministerio del Interior de Chile, Santiago.

Tapia, Jorge (2003), "Descentralización, diplomacia y paradiplomacia en la época de la globalización”, Estudios Fronterizos, 1: 25-47.

Vieira, Edgar (2005), "Evolución de las teorías sobre integración en el contexto de las teorías de relaciones internacionales”, Papel Político, 18: 235-290.

Viotti, Paul y Mark Kauppi, (1999), International Relations Theory. Realism, Pluralism, Globalism, and Beyond, Allyn and Bacon, Boston.

Waltz, Kenneth. (1979), Theory of International Politics, McGraw-Hill, Nueva York.

Wendt, Alexander (2005), "La anarquía es lo que los Estados hacen de ella". Revista Académica de Relaciones Internacionales, núm. 1.

Wendt, Alexander (1999), Social Theory of International Politics, Cambridge University, Cambridge.

Wilhelmy, Manfred (1979), "Hacia un análisis de la política exterior chilena contemporánea”, Estudios Internacionales, 48: 440-471.

Wilhelmy, Manfred y Roberto Durán (2003), "Los principales rasgos de la política exterior chilena entre 1973 y el 2000", Revista de Ciencia Política, 23(2): 273286.

Zacher, Mark y Richard Matthew (1995), “International Theory: Common Threads Divergent Strands, en C. Kegley (ed.), Controversies in International Relations Theory: Realism and Neoliberal Challenge, St. Martin's Press, Nueva York.

Recibido el 3 de enero de 2011 Segunda versión recibida el 2 de octubre de 2011 Aprobado el 10 de octubre de 2011 